\title{
Relationship of lycopene intake and consumption of tomato products to incident CVD
}

\author{
Paul F. Jacques ${ }^{1 *}$, Asya Lyass ${ }^{2,3}$, Joseph M. Massaro ${ }^{4}$, Ramachandran S. Vasan ${ }^{2,5}$ \\ and Ralph B. D'Agostino $\mathrm{Sr}^{2,3}$ \\ ${ }^{1}$ Jean Mayer US Department of Agriculture Human Nutrition Research Center on Aging, Tufts University, \\ 711 Washington Street, Boston, MA 02111, USA \\ ${ }^{2}$ NHLBI Framingham Heart Study, 73 Mount Wayte Avenue, Framingham, MA 01702, USA \\ ${ }^{3}$ Department of Mathematics and Statistics, College of Arts and Sciences, Boston University, 111 Cummington Street, Boston, \\ MA 02215, USA \\ ${ }^{4}$ Department of Biostatistics, School of Public Health, Boston University, 715 Albany Street, Boston, MA O2118, USA \\ ${ }^{5}$ Department of Medicine, School of Medicine, Boston University, 761 Harrison Avenue, Boston, MA O2118, USA \\ (Submitted 17 February 2012 - Final revision received 16 October 2012 - Accepted 22 October 2012 - First published online 15 January 2013)
}

\begin{abstract}
Evidence for cardioprotective effects of lycopene is inconsistent. Studies of circulating lycopene generally report inverse associations with CVD risk, but studies based on lycopene intake do not. The failure of dietary studies to support the findings based on biomarkers may be due in part to misclassification of lycopene intakes. To address this potential misclassification, we used repeated measures of intake obtained over 10 years to characterise the relationship between lycopene intake and the incidence of CVD ( $n$ 314), CHD ( $n$ 171) and stroke ( $n$ 99) in the Framingham Offspring Study. Hazard ratios (HR) for incident outcomes were derived from Cox proportional hazards regression models using logarithmically transformed lycopene intake adjusted for CVD risk factors and correlates of lycopene intake. HR were interpreted as the increased risk for a $2 \cdot 7$-fold difference in lycopene intake, a difference approximately equal to its interquartile range. Using an average of three intake measures with a 9-year follow-up, lycopene intake was inversely associated with CVD incidence (HR $0 \cdot 83,95 \%$ CI $0 \cdot 70,0 \cdot 98)$. Using an average of two intake measures and 11 years of follow-up, lycopene intake was inversely associated with CHD incidence (HR 0.74, 95\% CI 0.58, 0.94). Lycopene intake was unrelated to stroke incidence. The present study of lycopene intake and CVD provides supporting evidence for an inverse association between lycopene and CVD risk; however, additional research is needed to determine whether lycopene or other components of tomatoes, the major dietary source of lycopene, are responsible for the observed association.
\end{abstract}

\section{Key words: Lycopene: CVD: CHD: Stroke}

Individuals who consume higher amounts of vegetables and fruits consistently demonstrate lower risks of $\mathrm{CVD}^{(1-4)}$, but there is still much that we do not understand regarding the mechanism relating vegetable and fruit intake to CVD risk. Accumulating evidence from intervention trials suggests that the protective effect of vegetables and fruits may not necessarily be a consequence of their vitamin $\mathrm{E}$ and $\beta$-carotene ${ }^{(5)}$, whereas other research has suggested that lycopene, a dietary carotenoid obtained largely from tomato products, may provide cardioprotective benefits ${ }^{(6-9)}$. Many ${ }^{(6-9)}$, but not all ${ }^{(10-12)}$, prospective studies relating circulating lycopene concentrations and CVD risk have reported inverse associations, while studies based on dietary intake have generally failed to detect significant independent associations between lycopene and CVD risk $^{(13-17)}$. The reason for the failure of dietary studies to support the findings based on biomarkers is not known but may be due in part to misclassification of lycopene intakes.

To address the potential misclassification, we used repeated measures of intake obtained over 10 years to characterise the relationship between the intake of lycopene and tomato-based products and the incidence of CVD, CHD and stroke.

\section{Research design and methods}

Population description

The Framingham Heart Study began in 1948 with the enrolment of 5209 adults aged 28-62 years residing in Framingham,

Abbreviation: HR, hazard ratio.

*Corresponding author: P. F. Jacques, fax +1 6175563344 , email paul.jacques@tufts.edu 
a town west of Boston, $\mathrm{MA}^{(18)}$, and has continued for over 60 years, with the survivors returning every 2 years for a physical examination and to complete a series of questionnaires, laboratory and cardiovascular tests. By 1971, the original cohort included 1644 husband-wife pairs and 378 individuals who had developed CVD. The offspring of these subjects and the offspring's spouses were invited to participate, and 5135 of the 6838 eligible individuals participated in the first Framingham Offspring Study examination ${ }^{(19)}$. The Framingham Offspring cohort undergoes repeat examination approximately every 3-4 years. For the present study, we used data derived from the fifth, sixth and seventh study examinations, which spanned 10 years (1991-2001), with follow-up for incident CVD through to 2008.

The present study was conducted according to the guidelines laid down in the Declaration of Helsinki, and all procedures involving human participants were approved by the Boston University Medical Center Institutional Review Board. Written informed consent was obtained from all participants. The present study was approved by the Tufts Medical Center Institutional Review Board.

\section{Dietary assessment}

Dietary intakes were assessed using the Harvard semi-quantitative $\mathrm{FFQ}^{(20,21)}$ at the fifth, sixth and seventh examinations. The FFQ consists of a list of foods with a standard serving size and a selection of nine frequency categories ranging from never or $<1$ serving/month to $\geq 6$ servings/d. Participants were asked to report their frequency of consumption of each food item during the past year. Participants could also add up to three additional foods that are important components of their diets but are not listed on the questionnaire. Information on nutrient supplement use was also obtained by the FFQ. Dietary information was judged as unreliable and excluded from further study if reported energy intakes were $<2511 \mathrm{~kJ} / \mathrm{d}(600 \mathrm{kcal} / \mathrm{d})$ or $>16743 \mathrm{~kJ} / \mathrm{d}(4000 \mathrm{kcal} / \mathrm{d})$ for women and $>17580 \mathrm{~kJ} / \mathrm{d}(4200 \mathrm{kcal} / \mathrm{d})$ for men or if more than twelve food items were left blank.

Lycopene intake from food sources and multivitamin supplements was used to calculate lycopene intake. Ketchup, a relatively concentrated source of lycopene, was not listed as a standard food item on the FFQ, but participants had the option of listing it as an additional food. We also created a measure of tomato product consumption as the sum of tomatoes, tomato juice, tomato sauce and pizza consumption measured as servings per week.

Lycopene intake from the FFQ has been previously validated against plasma lycopene concentrations ${ }^{(22)}$ and intake of tomatoes has been validated using repeated diet records ${ }^{(23,24)}$. Correlations between lycopene intake based on the FFQ and plasma lycopene were 0.47 and 0.21 for non-smoking men and women, respectively. The lower correlation in women was believed to be a result of hormonal factors affecting circulating lycopene concentrations and not the result of a difference in the ability of the FFQ to capture the lycopene intake in men and women ${ }^{(22,25)}$. The correlation between the intake of tomatoes based on the FFQ and four
$7 \mathrm{~d}$ diet records for the corresponding time period was 0.73 in women ${ }^{(24)}$, while the correlation in men based on two $7 \mathrm{~d}$ diet records was $0 \cdot 71^{(23)}$.

\section{Ascertainment of CVD events and deaths}

All participants are under continuous surveillance for the occurrence of CVD events and death. Hospitalisation records and physician office visit records are obtained and reviewed by a committee of three experienced investigators. For each death, the same committee assigns an underlying cause of death based on Framingham Heart Study records, hospitalisation records and, when available, autopsy results ${ }^{(26)}$. Criteria for the diagnoses of cardiovascular events have been described elsewhere ${ }^{(27)}$. Incident CVD includes CHD (recognised or unrecognised myocardial infarction, angina pectoris, coronary insufficiency and CHD death), cerebrovascular disease (stroke and transient ischaemic attack), congestive heart failure (by Framingham criteria) and peripheral vascular disease (intermittent claudication). An unrecognised myocardial infarction was considered to have occurred if there was electrocardiographic evidence of significant loss of $\mathrm{R}$ waves or appearance of pathologic $Q$ waves on serial tracings in the absence of a clinically recognised event. Sudden death, defined as death occurring within $1 \mathrm{~h}$ of the onset of symptoms, is attributed to CHD unless another cause is apparent $^{(26)}$. Stroke was defined as an acute-onset focal neurological deficit of presumed vascular origin persisting for $\geq 24 \mathrm{~h}$. Transient ischaemic attack was defined as an episode of rapid-onset focal neurological dysfunction attributed to focal cerebral ischaemia with resolution within $24 \mathrm{~h}$. All cerebrovascular events were adjudicated by a panel of two neurologists ${ }^{(28)}$. For intermittent claudication, a physicianadministered standardised questionnaire was used to elicit subjective symptoms of calf discomfort with exertion that occurred sooner with uphill or fast-paced walking and was alleviated with rest. All suspected claudication events were verified independently by a second physician examiner.

\section{Assessment of covariates}

Covariates used in our analyses included sex, age, systolic blood pressure, total cholesterol, total cholesterol:HDL ratio, BMI, current smoking status, number of packs smoked per d, hypertension treatment, prevalent diabetes and dietary intakes of energy, saturated fat, $\beta$-carotene, flavonols, vitamin $\mathrm{C}$ and vitamin E. Medical history (e.g. medication use) and lifestyle activities (e.g. smoking history) were assessed during a standardised medical examination and interview. Height and weight were measured with the participant standing, shoes off and wearing only a hospital gown. BMI was calculated as body weight in $\mathrm{kg}$ divided by the square of height in $\mathrm{m}$. Sitting blood pressure was measured twice on each participant after a 5 min rest using a random-zero sphygmomanometer, and two readings were averaged for the analyses. Fasting ( $\geq 8 \mathrm{~h}$ ) blood samples were drawn for assessing lipid levels. Plasma total cholesterol was measured by an enzymatic method $^{(29)}$, and HDL-cholesterol was measured after 
dextran-magnesium precipitation ${ }^{(30)}$. Diabetes was defined as a plasma glucose level $\geq 7.0 \mathrm{mmol} / \mathrm{l}$ (fasting) or $\geq 11 \cdot 1 \mathrm{mmol} / 1$ (non-fasting), or use of hypoglycaemic drug therapy. We included $\beta$-carotene, flavonols, vitamin $\mathrm{C}$ and vitamin $\mathrm{E}$ as covariates not only as markers of vegetable and fruit consumption but also because these represent potential bioactive compounds found in varying quantities in tomatoes and other lycopene-containing foods.

\section{Statistical methods}

Our primary exposure was the natural logarithm of lycopene intake. Our primary outcome was incident CVD. Secondary analyses considered the intake of tomatoes and tomatobased products as an additional exposure, and incident CHD and stroke as outcomes. All analyses were performed using SAS version 9.2 (SAS Institute, Inc.). A $P$ value $<0.05$ was considered to be statistically significant.

Hazard ratios (HR) derived from Cox proportional hazards regression models (SAS PROC PHREG) were used to characterise the prospective associations between lycopene and tomato product intakes and the risk of CVD. Because lycopene intake was transformed using a natural logarithm, the HR is interpreted as the relative risk for a $2 \cdot 7$-fold difference (i.e. a one unit difference on the natural logarithmic scale) in lycopene intake. The $2 \cdot 7$-fold difference represents a reasonable intake difference in this population. It is similar to the ratio between the 75 th and 25 th percentiles of lycopene intake assessed at the fifth, sixth and seventh examinations, which ranged from $2 \cdot 4$ to $2 \cdot 6$.

In an attempt to minimise misclassification of lycopene and tomato product intake data, we considered two different exposures based on an average of intakes from the fifth and sixth examinations, and the fifth, sixth and seventh examinations. For each of these exposures, we initiated follow-up for CVD events at the date of the last examination included in the averaged intake. For example, follow-up for the averaged intake for the fifth and sixth examinations started at the date of the examination for participants at their sixth examination. Thus, the sixth examination served as baseline for the averaged intakes from the fifth and sixth examinations, and the seventh examination as baseline for the averaged intakes from the fifth, sixth and seventh examinations.

We considered three different models based on the inclusion of covariates: (1) an age- and sex-adjusted model (model 1); (2) a model with additional adjustment for systolic blood pressure, total cholesterol, total cholesterol:HDL ratio, BMI, smoking, number of packs per $\mathrm{d}$, hypertension treatment, prevalent diabetes, saturated fat intake and energy intake (model 2); (3) a model with additional adjustment for dietary factors including $\beta$-carotene, vitamin $\mathrm{C}$, vitamin $\mathrm{E}$ and flavonol intakes (model 3). Covariates, similar to the dietary data, reflect average values at the respective baseline examinations for the two follow-up periods.

To assess the validity of the proportional hazards assumption, we introduced interaction terms between follow-up time and our exposures, and use likelihood ratio tests to assess evidence for departures from this assumption.
There was no evidence that this assumption was violated for any of these models. We also examine the potential for effect modification by sex and smoking, but there was no evidence of any statistical interactions.

The number of participants available for follow-up was dependent on the baseline examination. Participation at the baseline examination was the initial criterion for inclusion. Among those participating in the sixth examination, 412 participants were excluded because of existing CVD, 313 because of missing or invalid dietary data and 131 because of missing covariate data, leaving 2667 participants for follow-up. At the seventh examination, 479 participants were excluded because of existing CVD, 406 because of missing or invalid dietary data and 121 because of missing covariate data, leaving 2525 participants for follow-up.

\section{Results}

The average age and BMI at the fifth examination were 54 years and $27.2 \mathrm{~kg} / \mathrm{m}^{2}$. Of the participants, $56 \%$ were women, $18 \%$ were current smokers, and 16 and $5 \%$ had a history of hypertension and diabetes, respectively. The mean lycopene intake was $7.6,7.9$ and $8.0 \mathrm{mg} / \mathrm{d}$ at the fifth, sixth and seventh examinations, and the mean consumption of tomatoes and tomato-based products was $4 \cdot 4,4.4$ and 4.6 servings/week at these three examinations.

The main contributors to lycopene intake at the fifth study examination were tomato sauce (46.4\%), pizza (23.6\%), fresh and canned tomatoes (14.6\%), tomato juice $(6.0 \%)$, watermelon $(5.7 \%)$, and grapefruit and grapefruit juice (2.6\%). No other food item contributed more than $1 \%$. There was a modest shift in sources of lycopene by the seventh examination where tomato sauce remained the predominant contributor to lycopene intake ( $45 \cdot 1 \%)$, followed by fresh and canned tomatoes $(26 \cdot 1 \%)$, pizza (12.4\%), watermelon $(7.4 \%)$, tomato juice $(5.9 \%)$ and grapefruit and grapefruit juice $(2 \cdot 3 \%)$.

The median follow-up was approximately 11 years from the sixth examination and approximately 9 years from the seventh examination. Although there were no statistically significant associations observed between lycopene intake and CVD incidence using the average of two intake assessments from the fifth and sixth examinations (Table 1), we observed significant associations between lycopene intake based on the average of three measures from the fifth, sixth and seventh examinations and CVD after adjustment for CVD risk factors (model 2) and additional adjustment for dietary correlates of lycopene intake (model 3). Interpretation of the HR from these models is complicated by the natural logarithm transformation of lycopene intake. For example, the HR for model 3 based on the average intake from the fifth, sixth and seventh examinations (HR 0.83) would translate into a $17 \%$ lower incidence of CVD for a $2 \cdot 7$-fold difference in lycopene intake. The $2 \cdot 7$-fold difference is similar to the ratio between the 75 th and 25 th percentiles of lycopene intake assessed at the fifth, sixth and seventh examinations.

The evidence for a relationship between lycopene intake and overall CHD shows a slightly different pattern than CVD 
Table 1. Lycopene and tomato product intakes and incidence of CVD, CHD and stroke estimated from the hazard ratio (HR) among adult men and women from the Framingham Offspring cohort ${ }^{\star}$ (Hazard ratios and $95 \%$ confidence intervals)

\begin{tabular}{|c|c|c|c|c|c|c|c|c|c|c|c|c|}
\hline & \multicolumn{4}{|c|}{ CVD } & \multicolumn{4}{|c|}{$\mathrm{CHD}$} & \multicolumn{4}{|c|}{ Stroke } \\
\hline & No. of events & $\mathrm{HR}$ & $95 \% \mathrm{Cl}$ & $P$ & No. of events & $\mathrm{HR}$ & $95 \% \mathrm{Cl}$ & $P$ & No. of events & $\mathrm{HR}$ & $95 \% \mathrm{Cl}$ & $P$ \\
\hline \multicolumn{13}{|l|}{ Lycopene intake $\dagger$} \\
\hline Examinations 5 and 6 average $\neq$ & 23573 person-years & & & & 23618 person-years & & & & 23609 person-years & & & \\
\hline Model $1 \S$ & 314 & $0.92 \|$ & $0.77,1.10$ & 0.39 & 171 & 0.77 & $0.61,0.96$ & 0.03 & 99 & 1.05 & $0.76,1.46$ & 0.76 \\
\hline Model 2ף & 314 & 0.87 & $0.72,1.05$ & 0.16 & 171 & 0.76 & $0.60,0.97$ & 0.03 & 99 & 1.08 & $0.75,1.55$ & 0.68 \\
\hline Model $3^{\star \star}$ & 314 & 0.86 & $0.71,1.05$ & $0 \cdot 13$ & 171 & 0.74 & $0.58,0.94$ & 0.01 & 99 & 1.07 & $0.74,1.54$ & 0.74 \\
\hline Examinations 5, 6 and 7 average $\dagger \dagger$ & 15858 person-years & & & & 15870 person-years & & & & 15880 person-years & & & \\
\hline Model 1 & 219 & 0.87 & $0.73,1.04$ & 0.14 & 115 & 0.86 & $0.68,1.08$ & 0.20 & 66 & 0.92 & $0.65,1.32$ & 0.67 \\
\hline Model 2 & 219 & 0.82 & $0.70,0.97$ & 0.03 & 115 & 0.84 & $0.68,1.05$ & 0.13 & 66 & 0.83 & $0.58,1 \cdot 18$ & 0.31 \\
\hline Model 3 & 219 & 0.83 & $0.70,0.98$ & 0.03 & 115 & 0.84 & $0.67,1.03$ & $0 \cdot 10$ & 66 & 0.82 & $0.59,1.16$ & 0.27 \\
\hline \multicolumn{13}{|l|}{ Tomato product consumptionł‡ } \\
\hline Examinations 5 and 6 average & 23573 person-years & & & & 23618 person-years & & & & 23609 person-years & & & \\
\hline Model 1 & 314 & $0.98 \S \S$ & $0.94,1.02$ & 0.45 & 171 & 0.94 & $0.88,0.99$ & 0.04 & 99 & 1.02 & $0.95,1.08$ & 0.63 \\
\hline Model 2 & 314 & 0.98 & $0.94,1.02$ & 0.28 & 171 & 0.94 & $0.88,1.00$ & 0.05 & 99 & 1.02 & $0.96,1.09$ & 0.51 \\
\hline Model 3 & 314 & 0.97 & $0.93,1.02$ & 0.24 & 171 & 0.92 & $0.86,0.99$ & 0.02 & 99 & 1.02 & $0.96,1.10$ & 0.51 \\
\hline Examinations 5, 6 and 7 average & 15858 person-years & & & & 15870 person-years & & & & 15880 person-years & & & \\
\hline Model 1 & 219 & 0.97 & $0.916,1.022$ & 0.25 & 115 & 0.95 & $0.87,1.02$ & 0.16 & 66 & 1.02 & $0.93,1.11$ & 0.68 \\
\hline Model 2 & 219 & 0.94 & $0.887,0.999$ & 0.05 & 115 & 0.93 & $0.85,1.001$ & 0.08 & 66 & 1.00 & $0.91,1.10$ & 0.99 \\
\hline Model 3 & 219 & 0.94 & $0.878,0.995$ & 0.03 & 115 & 0.90 & $0.83,0.99$ & 0.03 & 66 & 0.99 & $0.90,1.10$ & 0.91 \\
\hline
\end{tabular}

*At the fifth study examination, the Framingham Offspring cohort participants had a mean age of 54 years (range $26-79$ years) and a mean BMI of $27 \cdot 2 \mathrm{~kg} / \mathrm{m}^{2}\left(\mathrm{range} 14-54 \mathrm{~kg} / \mathrm{m}^{2}\right)$. Of the participants, $56 \%$ were women, $18 \%$ were current smokers, and 16 and $5 \%$ had a history of hypertension and diabetes, respectively.

† Mean lycopene intake was $7.6,7.9$ and $8.0 \mathrm{mg} / \mathrm{d}$ at the fifth, sixth and seventh examinations, respectively.

Ғ Intake is the average of intakes assessed at the Framingham Offspring cohort fifth and sixth examinations and follow-up time is counted from the date of the sixth examination.

$\S$ Model 1: adjusted for age and sex.

|| Lycopene intake was transformed using a natural logarithm. Consequently, the HR are interpreted as the relative risk for a 2.7-fold difference (i.e. a one-unit difference on the natural logarithmic scale) in lycopene intake.

I Model 2: adjusted for age, sex, systolic blood pressure, total cholesterol, total cholesterol:HDL ratio, BMI, smoking, number of packs per d, hypertension treatment, diabetes, saturated fat intake and energy intake.

${ }_{* \star}$ Model 3: adjusted for variables in model 2 plus $\beta$-carotene, flavonol, vitamin $C$ and vitamin $E$ intakes.

t† Intake is the average of intakes assessed at the Framingham Offspring cohort fifth, sixth and seventh examinations and follow-up time is counted from the date of the seventh examination.

¥¥ Mean consumption of tomatoes and tomato-based products was 4.4, 4.4 and $4 \cdot 6$ servings/week at the fifth, sixth and seventh examinations, respectively.

$\S \S$ The HR reflect the relative risk for a one serving per d difference in tomato product consumption. 
(Table 1). We observed that increasing intake of lycopene based on the average of intakes from the fifth and sixth examinations was associated with a significantly lower risk of CHD, but the association between lycopene intake and CHD based on the average of the fifth, sixth and seventh examinations and the shorter follow-up period was not statistically significant. The association based on the average of the sixth and seventh examinations remained significant after multivariate adjustment. The HR of 0.74 for model 3 would translate into a $26 \%$ lower incidence of CHD for a $2 \cdot 7$-fold difference in lycopene intake. No significant associations were observed between lycopene intake and stroke (Table 1).

The relationship between tomato and tomato product consumption and CVD and CHD was very similar to that seen with lycopene consumption (Table 1), except that the relationship between tomato product consumption and CHD was also apparent when using the intakes averaged from three examinations. The tomato products included in this analysis accounted for approximately $90 \%$ of lycopene intake. As with lycopene intake, we observed no associations between tomato and tomato product consumption and the risk of stroke.

\section{Discussion}

These present findings demonstrate an inverse association between lycopene intake and the risk of $\mathrm{CHD}$, but not stroke, and, along with evidence from earlier studies relating serum lycopene to the risk of $\mathrm{CVD}^{(6-9)}$ and carotid artery intima-media thickness ${ }^{(31,32)}$, provide support for the hypothesis that higher lycopene is associated with lower CVD risk. In contrast, the previous studies of dietary lycopene and CVD risk provided little evidence of any association ${ }^{(13-17)}$. One of these studies based on male smokers in the Alpha-Tocopherol, Beta-Carotene Cancer Prevention Trial reported an inverse association with two subtypes of stroke (cerebral infarction and intracerebral haemorrhage) but lycopene intakes in this cohort were very low (median intake of $0.59 \mathrm{mg} / \mathrm{d})^{(16)}$, complicating the interpretation of this finding. There was no significant association observed between lycopene intake and CVD risk in the Women's Health Initiative; however, in agreement with the observations from the present study, there was a marginally significant $31 \%$ reduction in the risk of CHD associated with an approximately 5-fold difference in lycopene intakes from the lowest to the highest intake quintile categories ${ }^{(14)}$. The failure to detect a significant association may be the consequence of potential bias introduced by misclassification of exposure because lycopene intake was assessed using one baseline measure, and then participants were followed for a median follow-up of $7 \cdot 2$ years. Misclassification is a less likely explanation for the failure to observe significant associations in two other studies as lycopene intake was updated using cumulative averages during the follow-up ${ }^{(13,15)}$. The final study to examine the relationship between dietary lycopene and CVD is a retrospective casecontrol study, which is subject to more sources of bias, including misclassification of lycopene intake as intake was based on a single assessment ${ }^{(17)}$.
We also observed inverse associations between the consumption of tomatoes and tomato products and CVD and CHD incidence. The aforementioned Women's Health Initiative study, which found no significant association between lycopene intake and CVD risk, reported significant inverse associations between the consumption of tomato products and CHD and total $\mathrm{CVD}^{(14)}$.

We did not observe any evidence of an association between stroke incidence and either lycopene intake or tomato product consumption. However, given the relatively small number of events, particularly for the analyses based on the average of three intake measures, it is possible that the failure to observe any relationship with stroke was a consequence of inadequate statistical power.

Bioavailability and absorption of lycopene are relatively low and depend on the processing and preparation of lycopenecontaining foods and on the fat content of the meal in which lycopene is consumed ${ }^{(33-36)}$. Low and variable bioavailability and absorption will result in greater misclassification of actual lycopene exposures based on intake, making it more difficult to establish the relationships between intake and health outcomes. Although the use of multiple measures of lycopene intake would not limit misclassification associated with bioavailability, our ability to characterise lycopene intake using repeated measures of intake may have allowed us to limit variability from other sources, increasing our ability to detect associations with CVD risk.

In addition to the use of multiple measures of dietary intake, the present study has other strengths, most notably the use of the Framingham Heart Study Offspring cohort, a pre-eminent longitudinal study of CVD. The Framingham Heart Study provides tremendous advantages for characterisation of CVD outcomes and important risk factor information. The fact that ketchup was not listed as a standard item of the FFQ is a potential limitation of the present study. Although participants could chose to include ketchup as an additional food item, it is likely that many participants did not report ketchup consumption. Ketchup is a concentrated source of lycopene that contains approximately the same amount of lycopene per unit weight as tomato sauce ${ }^{(37)}$. However, because of its small serving size relative to other important lycopene food sources (typically $<15 \%$ by weight), the contribution of lycopene per serving is relatively small, so the misclassification of lycopene intake based on the incomplete ascertainment of ketchup intake should be modest overall, although it could be substantial for individuals who frequently consume ketchup. The FFQ also did not capture lycopene from single nutrient supplements, although we were able to measure lycopene included in multivitamins. As with ketchup, the average contribution from lycopene supplements would probably be small in this population but we would substantially misclassify lycopene intake for anyone who used these supplements. However, the misclassification associated with both ketchup and lycopene supplements would tend to weaken any observed association between lycopene and tomato intake and CVD risk. Finally, we did not adjust for overall vegetable and fruit intake because these are the dietary sources of lycopene, and adjusting for all fruits and vegetables (including 
those that contribute to lycopene intake) may result in some degree of over-adjustment. Instead, we chose to adjust for various bioactive compounds found in vegetables and fruits that may have the potential to confound the relationship between lycopene intake and CVD.

Evidence linking lycopene intake to intermediate CVD risk factors is somewhat limited in both experimental and human studies. In vitro and animal studies have suggested that lycopene can inhibit the production of reactive oxygen species, inflammation and platelet aggregation, reduce lipid peroxides and decrease total and LDL-cholesterol levels ${ }^{(38)}$. A metaanalysis of small human intervention studies has reported that lycopene intakes of approximately $25 \mathrm{mg} / \mathrm{d}$ resulted in a $10 \%$ reduction in LDL-cholesterol relative to subjects on lycopene-free diets, and a small number of interventions between 4.5 and $15 \mathrm{mg} / \mathrm{d}$ of lycopene resulted in a significant $5 \mathrm{mmHg}$ drop in systolic blood pressure ${ }^{(39)}$. A recent observational study was also able to demonstrate an inverse association between tomato product consumption and cholesterol concentrations $^{(40)}$. Serum LDL conjugated dienes, a marker of lipid oxidation, was also related to plasma lycopene in women but not in men ${ }^{(41)}$.

The present study of lycopene and incident CVD adds to the accumulating evidence that lycopene is related to CVD risk. However, tomatoes and tomato-based products are by far the most important dietary sources of lycopene in observational studies, and most human lycopene trials are performed using tomato-based interventions. Thus, it is difficult to separate out the potential lycopene contribution to cardiovascular health from the overall contribution from tomato products and their other components. For example, tomatoes have been identified as a source of 9-oxo-10,12-octadecadienoic acid and its isomers, which are important oxylipins synthesised in plants that may act as PPAR $\alpha$ agonists affecting lipid metabolism ${ }^{(42)}$. Tomatoes have also been identified as a source of natural salicylates $(3 \cdot 1 \mu \mathrm{g} / 100 \mathrm{~g})$, although it is not known whether the dietary levels of salicylates provided by tomato products would be sufficient to produce any cardiovascular benefits ${ }^{(43)}$. Additional research is needed to sort out the potential benefits of tomatoes and their phytochemical components on CVD risk.

\section{Acknowledgements}

This study was supported by the European Union LYCOCARD Project through grant no. B514-09057 from the Ministry of Education and Cultural Affairs of the Free State of Thuringia, Germany, and by the National Institutes of Health/National Heart, Lung, and Blood Institute (contract N01-HC-25,195) and the United States Department of Agriculture (agreement no. 58-1950-7-707). None of the authors has a conflict of interest to declare. The authors' contributions were as follows: P. F. J., R. S. V. and R. B. D'A. contributed to the study design; J. M. M. provided the statistical guidance; A. L. performed the statistical analyses; P. F. J. drafted the manuscript; P. F. J., R. S. V., R. B. D'A., J. M. M. and A. L. contributed to the interpretation of the results and the editing of the manuscript.

\section{References}

1. He FJ, Nowson CA \& MacGregor GA (2006) Fruit and vegetable consumption and stroke: meta-analysis of cohort studies. Lancet 367, 320-326.

2. He FJ, Nowson CA, Lucas M, et al. (2007) Increased consumption of fruit and vegetables is related to a reduced risk of coronary heart disease: meta-analysis of cohort studies. J Hum Hypertens 21, 717-728.

3. Dauchet L, Amouyel P \& Dallongeville J (2005) Fruit and vegetable consumption and risk of stroke: a meta-analysis of cohort studies. Neurology 65, 1193-1197.

4. Dauchet L, Amouyel P, Hercberg S, et al. (2006) Fruit and vegetable consumption and risk of coronary heart disease: a meta-analysis of cohort studies. J Nutr 136, 2588-2593.

5. Bruckdorfer KR (2008) Antioxidants and CVD. Proc Nutr Soc 67, 214-222.

6. Rissanen TH, Voutilainen S, Nyyssonen K, et al. (2001) Low serum lycopene concentration is associated with an excess incidence of acute coronary events and stroke: the Kuopio Ischaemic Heart Disease Risk Factor Study. Br J Nutr 85, 749-754.

7. Sesso HD, Buring JE, Norkus EP, et al. (2004) Plasma lycopene, other carotenoids, and retinol and the risk of cardiovascular disease in women. Am J Clin Nutr 79, 47-53.

8. Hak AE, Ma J, Powell CB, et al. (2004) Prospective study of plasma carotenoids and tocopherols in relation to risk of ischemic stroke. Stroke 35, 1584-1588.

9. Ito Y, Kurata M, Suzuki K, et al. (2006) Cardiovascular disease mortality and serum carotenoid levels: a Japanese population-based follow-up study. J Epidemiol 16, 154-160.

10. Sesso HD, Buring JE, Norkus EP, et al. (2005) Plasma lycopene, other carotenoids, and retinol and the risk of cardiovascular disease in men. Am J Clin Nutr 81, 990-997.

11. Hak AE, Stampfer MJ, Campos H, et al. (2003) Plasma carotenoids and tocopherols and risk of myocardial infarction in a low-risk population of US male physicians. Circulation 108, 802-807.

12. Street DA, Comstock GW, Salkeld RM, et al. (1994) Serum antioxidants and myocardial infarction. Are low levels of carotenoids and alpha-tocopherol risk factors for myocardial infarction? Circulation 90, 1154-1161.

13. Osganian SK, Stampfer MJ, Rimm E, et al. (2003) Dietary carotenoids and risk of coronary artery disease in women. Am J Clin Nutr 77, 1390-1399.

14. Sesso HD, Liu S, Gaziano JM, et al. (2003) Dietary lycopene, tomato-based food products and cardiovascular disease in women. J Nutr 133, 2336-2341.

15. Ascherio A, Rimm EB, Hernan MA, et al. (1999) Relation of consumption of vitamin $\mathrm{E}$, vitamin $\mathrm{C}$, and carotenoids to risk for stroke among men in the United States. Ann Intern Med 130, 963-970.

16. Hirvonen T, Virtamo J, Korhonen P, et al. (2000) Intake of flavonoids, carotenoids, vitamins $\mathrm{C}$ and $\mathrm{E}$, and risk of stroke in male smokers. Stroke 31, 2301-2306.

17. Tavani A, Gallus S, Negri E, et al. (2006) Dietary intake of carotenoids and retinol and the risk of acute myocardial infarction in Italy. Free Radic Res 40, 659-664.

18. Dawber TR \& Kannel WB (1958) An epidemiologic study of heart disease: the Framingham study. Nutr Rev 16, 1-4.

19. Feinleib M, Kannel WB, Garrison RJ, et al. (1975) The Framingham Offspring Study. Design and preliminary data. Prev Med 4, 518-525.

20. Rimm EB, Giovannucci EL, Stampfer MJ, et al. (1992) Reproducibility and validity of an expanded self-administered semiquantitative food frequency questionnaire among male 
health professionals. Am J Epidemiol 135, 1114-1126 (discussion 1127-1136).

21. Willett WC, Sampson L, Stampfer MJ, et al. (1985) Reproducibility and validity of a semiquantitative food frequency questionnaire. Am J Epidemiol 122, 51-65.

22. Michaud DS, Giovannucci EL, Ascherio A, et al. (1998) Associations of plasma carotenoid concentrations and dietary intake of specific carotenoids in samples of two prospective cohort studies using a new carotenoid database. Cancer Epidemiol Biomarkers Prev 7, 283-290.

23. Feskanich D, Rimm EB, Giovannucci EL, et al. (1993) Reproducibility and validity of food intake measurements from a semiquantitative food frequency questionnaire. $J \mathrm{Am}$ Diet Assoc 93, 790-796.

24. Salvini S, Hunter DJ, Sampson L, et al. (1989) Food-based validation of a dietary questionnaire: the effects of weekto-week variation in food consumption. Int J Epidemiol 18, 858-867.

25. Forman MR, Beecher GR, Muesing R, et al. (1996) The fluctuation of plasma carotenoid concentrations by phase of the menstrual cycle: a controlled diet study. Am J Clin Nutr 64, $559-565$.

26. Lloyd-Jones DM, Martin DO, Larson MG, et al. (1998) Accuracy of death certificates for coding coronary heart disease as the cause of death. Ann Intern Med 129, 1020-1026.

27. Kannel WB, Wolf PA \& Garrison RJ (1987) Some Risk Factors Related to the Annual Incidence of Cardiovascular Disease and Death in Pooled Repeated Biennial Measurements. Framingham Heart Study, 30 year Follow-up. Bethesda, MD: US Department of Health and Human Services.

28. Carandang R, Seshadri S, Beiser A, et al. (2006) Trends in incidence, lifetime risk, severity, and 30-day mortality of stroke over the past 50 years. JAMA 296, 2939-2946.

29. McNamara JR \& Schaefer EJ (1987) Automated enzymatic standardized lipid analyses for plasma and lipoprotein fractions. Clin Chim Acta 166, 1-8.

30. Warnick GR, Benderson J \& Albers JJ (1982) Dextran sulfate$\mathrm{Mg}^{2+}$ precipitation procedure for quantitation of highdensity-lipoprotein cholesterol. Clin Chem 28, 1379-1388.

31. Rissanen T, Voutilainen S, Nyyssonen K, et al. (2000) Low plasma lycopene concentration is associated with increased intima-media thickness of the carotid artery wall. Arterioscler Thromb Vasc Biol 20, 2677-2681.

32. Rissanen TH, Voutilainen S, Nyyssonen K, et al. (2003) Serum lycopene concentrations and carotid atherosclerosis: the Kuopio Ischaemic Heart Disease Risk Factor Study. Am J Clin Nutr 77, 133-138.

33. Rao AV \& Rao LG (2007) Carotenoids and human health Pharmacol Res 55, 207-216.

34. Arab L \& Steck S (2000) Lycopene and cardiovascular disease. Am J Clin Nutr 71, 1691S-1695S (discussion 1696S-1697S)

35. Tang G, Ferreira AL, Grusak MA, et al. (2005) Bioavailability of synthetic and biosynthetic deuterated lycopene in humans. J Nutr Biochem 16, 229-235.

36. Brown MJ, Ferruzzi MG, Nguyen ML, et al. (2004) Carotenoid bioavailability is higher from salads ingested with full-fat than with fat-reduced salad dressings as measured with electrochemical detection. Am J Clin Nutr 80, 396-403.

37. Holden JM, Eldridge AL, Beecher GR, et al. (1999) Carotenoid content of U.S. foods: an update of the database. J Food Comp Anal 12, 169-196.

38. Bohm V (2012) Lycopene and heart health. Mol Nutr Food Res 56, 296-303.

39. Ried K \& Fakler P (2011) Protective effect of lycopene on serum cholesterol and blood pressure: meta-analyses of intervention trials. Maturitas 68, 299-310.

40. Sesso HD, Wang L, Ridker PM, et al. (2012) Tomato-based food products are related to clinically modest improvements in selected coronary biomarkers in women. $J$ Nutr 142, 326-333.

41. Karppi J, Nurmi T, Kurl S, et al. (2010) Lycopene, lutein and beta-carotene as determinants of LDL conjugated dienes in serum. Atherosclerosis 209, 565-572.

42. Kim YI, Hirai S, Takahashi H, et al. (2011) 9-oxo-10(E),12(E)Octadecadienoic acid derived from tomato is a potent PPAR alpha agonist to decrease triglyceride accumulation in mouse primary hepatocytes. Mol Nutr Food Res 55, 585-593.

43. Duthie GG \& Wood AD (2011) Natural salicylates: foods, functions and disease prevention. Food Funct 2, 515-520 